\title{
Facile synthesis of nitrogen-doped graphene via low-temperature pyrolysis: The effects of precursors and annealing ambience on metal-free catalytic oxidation
}

Degang $\mathrm{Li}^{1}$, Xiaoguang Duan ${ }^{2 *}$, Hongqi $\mathrm{Sun}^{3}$, Jian Kang ${ }^{2}$, Huayang Zhang ${ }^{2}$, Moses O. Tade ${ }^{2}$, Shaobin Wang ${ }^{2, *}$

${ }^{1}$ School of Chemical Engineering, Shandong University of Technology, Zibo 255049, China

2 Department of Chemical Engineering, Curtin University, GPO Box U1987, WA 6845, Australia

${ }^{3}$ School of Engineering, Edith Cowan University, Joondalup, WA 6027, Australia.

*Corresponding authors:

Tel: +61 89266 3776. E-mail: shaobin.wang@curtin.edu.au (Shaobin Wang)

Tel: +61 89266 5410. E-mail: xiaoguang.duan@curtin.edu.au (Xiaoguang Duan) 


\begin{abstract}
A green and facile protocol of thermal treatment of graphene oxide (GO) with urea was adopted to synthesize nitrogen-doped graphene (NG-Urea-air) at a low temperature $\left(350{ }^{\circ} \mathrm{C}\right)$ in the static air. The resulting sample exhibited outstanding catalytic performance to activate peroxymonosulfate (PMS) toward organic degradation. The NG-Urea-air induced 49.7- and 11.5-fold enhancement over GO and pristine reduced graphene oxide (rGO-air). Moreover, the influences of nitrogen precursors including organic chemicals (urea, cyanamide, and melamine) and inorganic salts (ammonium nitrate and ammonium chloride) were investigated, and urea was demonstrated to be the best precursor for synthesizing $\mathrm{N}$-doped graphene with a relative high doping level (18.7 at.\%). The classical radical quenching and advanced in situ electron paramagnetic resonance (EPR) technology revealed that the outstanding oxidative effectiveness of PMS/NG-Urea-air system was originated from the nonradical oxidation pathway, in which PMS was activated by the positively charged carbon domains next to nitrogen atoms and the phenol was oxidized simultaneously on the carbon network via rapid charge transfer. Meanwhile, singlet oxygen and radicals may also partially contribute to the complete phenol degradation. This study facilitates a fundamental investigation of heteroatom doping progress during thermal treatment and sheds light on the insights into carbocatalysis in environmental remediation.
\end{abstract}

Keywords: Catalytic oxidation; nitrogen-doped graphene; precursors; singlet oxygen, peroxymonosulfate. 


\section{Introduction}

Since A.K. Geim uncovered the existence of single-layered graphite, namely graphene, with carbon atoms perfectly packed into the honeycomb lattice in a uniform $\mathrm{sp}^{2}$-hybridized configuration, graphene-based materials have opened up a new avenue to the world of materials science, nanotechnology, and chemical/energy conversions [1, 2]. Versatile approaches, including chemical vapor deposition [3], epitaxial growth [4], and exfoliation of graphite [5], have been developed to synthesize graphene with desired physicochemical and electronic properties for wide applications. The exfoliation of graphite, the stacked layers of graphene, has attracted extensive interests because of the cheap and abundant carbon source and mild synthesis conditions. Several protocols of exfoliation have been developed such as micromechanical exfoliation [6], electrochemical exfoliation [7], and chemical exfoliation [8, 9]. Among these methods, the Hummers' approach was most widely adopted to synthesize single or few-layered chemically exfoliated graphene oxide (GO), followed by post-processing with thermal-treatment or chemical reduction to achieve a better reductive degree of graphene, also known as reduced graphene oxide (rGO) [10-12]. Moreover, GO with sufficient oxygen functional groups, smaller particle sizes, and massive exposed edging sites offers a promising matrix for surface modification, especially for heteroatom doping.

Introducing heteroatoms $(\mathrm{B}, \mathrm{N}, \mathrm{O}, \mathrm{P}, \mathrm{S}$, and so forth) into the carbon lattice can effectively disorientate the homogeneously conjugated electron network and modulate the surface properties by tweaking the charge distribution and spinning culture of the doped domains [13, 14]. Nitrogen-doped graphene has been intensively investigated in recent years for metal-free catalysis. Nitrogen is adjacent to carbon in the periodic table of elements. Nitrogen atom pocesses a similar atomic radius $(0.70 \AA)$ as carbon $(0.77 \AA)$ and a more negative electronegativity $\left(\chi_{\mathrm{N}}=3.04\right)$ than $\mathrm{C}\left(\chi_{\mathrm{C}}=2.55\right)$, making it easier to incorporate into the carbon 
basal plane via substitutional doping. $\mathrm{N}$ can reach a considerable doping level, meanwhile intrinsically altering the electron states of the graphene. Therefore, $\mathrm{N}$-doping has been demonstrated to be able to endow pristine graphene with impressively enhanced activity toward chemical reactions and catalysis [15-17]. Various N-rich substances such as ammonia, ammonium salts, and organic matters have been utilized as the nitrogen precursors. However, very little attention has been paid to the effects of different precursors and the mechanism of $\mathrm{N}$-doping process. Understanding the impacts of diverse precursors and the doping procedure will provide fundamental knowledge for the rational design of efficient carbocatalysts via elaborate nano-engineering.

In this study, we applied a facile strategy by direct treatment of GO and urea at a moderate temperature $\left(350^{\circ} \mathrm{C}\right)$. This protocol utilized cheap and green nitrogen precursors under mild conditions for synthesis of $\mathrm{N}$-doped graphene without intensive energy input and rigorous reaction requirements for the aforementioned micromechanical/electrochemical exfoliation and chemical vapor deposition (CVD) approaches. The derived carbocatalysts were utilized to activate peroxymonosulfate (PMS) for catalytic oxidation. The chemically modified graphene exhibited significantly improved activity than the pristine graphene. Moreover, the N-doping procedure was investigated by exploring the effects of carbon matrix (oxygen-rich/deficient carbon precursors), N-doping precursors (organic/inorganic nitrogen-containing substances), and annealing conditions (oxidative or non-oxidative ambience). The green and efficient carbocatalysts have demonstrated extraordinary potentials for activating various superoxides (e.g. peroxymonosulfate, persulfate, hydrogen peroxide, and ozone) for the oxidative removal of toxic pollutants in wastewater without any secondary contamination [18-21]. However, due to the complicated structure and surface chemistry of nanocarbons, the mechanism of carbocatalysis in metal-free oxidation remains ambiguous, leaving more blanks for mechanistic 
study. This investigation facilitates an in-depth study of PMS activation on graphene-based materials with the occurrence of the nonradical mechanism and evolution of singlet oxygen besides the traditional radical pathway. The findings of this study would unveil the principles of achieving desirable N-doping in graphene-based materials and will definitely contribute to carbon-based materials science, green environmental remediation, and metal-free catalysis technologies.

\section{Experimental}

\subsection{Materials preparation}

The chemicals in this study were purchased from Sigma-Aldrich, Australia with a reagent grade and used as received without further purification. Firstly, graphene oxide (GO) was prepared via a chemical exfoliation approach based-on a modified Hummers' method, and the detailed procedures can be found in a previous study [18]. The nitrogen doped graphene (NG) was synthesized by a facile pyrolysis of GO with nitrogen precursors. Briefly, GO $(0.5 \mathrm{~g})$ and urea $(0.60 \mathrm{~g})$ were dissolved in $30 \mathrm{~mL}$ ethanol and treated in an ultrasonic bath for $30 \mathrm{~min}$ to form a homogeneous solution. Then the mixture was heated on a hotplate at $60{ }^{\circ} \mathrm{C}$ to evaporate the solvent whilst keeping stirred. The obtained black mixture was ground finely, transferred to the muffle furnace in a crucible, and heated at $350{ }^{\circ} \mathrm{C}$ for $30 \mathrm{~min}$ in a static air with a heating rate of $5{ }^{\circ} \mathrm{C} / \mathrm{min}$. The obtained furry black powder was then washed with ethanol and deionized water several times and then dried in an oven at $60{ }^{\circ} \mathrm{C}$ overnight for further use. The products were ground and denoted as NG-Urea-air. Reduced graphene oxide (rGO-air) was prepared by the same procedure without the addition of nitrogen precursors. To investigate the effect of carbon sources, the GO was substituted by rGO-air in the aforementioned process and the product was marked as N-rGO-air. To investigated the effect of annealing environment, similar nanocarbon materials were prepared by treating the GO (or $\mathrm{rGO}$ )/urea mixture in a tubular 
furnace under $\mathrm{N}_{2}(60 \mathrm{~mL} / \mathrm{min})$, and the resulting products were named as $\mathrm{rGO}-\mathrm{N}_{2}$, NG-Urea$\mathrm{N}_{2}$, and N-rGO- $\mathrm{N}_{2}$. Besides, different $\mathrm{N}$-precursors were also investigated with other organic precursors such as melamine, cyanamide, and inorganic salts such as ammonium nitrate $\left(\mathrm{NH}_{4} \mathrm{NO}_{3}\right)$ and ammonium chloride $\left(\mathrm{NH}_{4} \mathrm{Cl}\right)$. The dosage of the precursors was calculated based on the containing of $0.02 \mathrm{~mol}$ nitrogen atoms in all the precursors. The $\mathrm{N}$-doped graphene from different $\mathrm{N}$-precursors were prepared in the same procedure as NG-Urea-air, and the products were denoted as NG-Melamine, NG-Cyanamide, $\mathrm{NG}-\mathrm{NH}_{4} \mathrm{NO}_{3}$, and $\mathrm{NG}-\mathrm{NH}_{4} \mathrm{Cl}$, respectively.

\subsection{Characterization of nanocarbons}

The structure and morphology of the nanocarbons was revealed by a Zeiss Neon FIBSEM. The elemental distribution of nitrogen-doped graphene from different precursors was monitored by the EDS elemental mapping in Figs. S1 - S5. FTIR spectra were acquired from a Bruker spectrometer. The BET surface area, nitrogen sorption isotherms, and pore diameter distributions were measured at $-196{ }^{\circ} \mathrm{C}$ on a TriStar II apparatus. The surface elemental information was probed with a Kratos X-ray photoelectron spectroscopy (XPS) instrument equipped with a monochromated $\mathrm{Al} \mathrm{K} \alpha \mathrm{X}$-ray gun.

\subsection{Catalytic oxidation procedure}

The catalytic performance of the nanocarbon catalysts was evaluated by PMS activation and oxidation of toxic organic contaminants. The aqueous reactions were carried out in a batch reactor with the organics, PMS, and carbocatalysts at the usage of 20 ppm organic chemicals, 6.5 mM PMS, and $0.4 \mathrm{~g} / \mathrm{L}$ carbocatalysts at $25^{\circ} \mathrm{C}$. The solution was withdrawn at set intervals and filtered to remove the solid catalyst, quenched by methanol, and analyzed on an ultra-high performance liquid chromatography (UHPLC, ThermoFisher Scientific) with an UltiMate ${ }^{\mathrm{TM}}$ 
3000 RSLCnano system. The generated singlet oxygen was captured by 2,2,6,6-tetramethyl-4piperidone (TMP) and tested on a Bruker electron paramagnetic resonance (EPR) instrument with the settings at following parameters: center field $3515 \mathrm{G}$, sweep width $100.0 \mathrm{G}$, power $20.0 \mathrm{~mW}$, sweeping time $30 \mathrm{~s}$, and scan number 2.

\section{Results and Discussion}

\subsection{Characterization of samples}

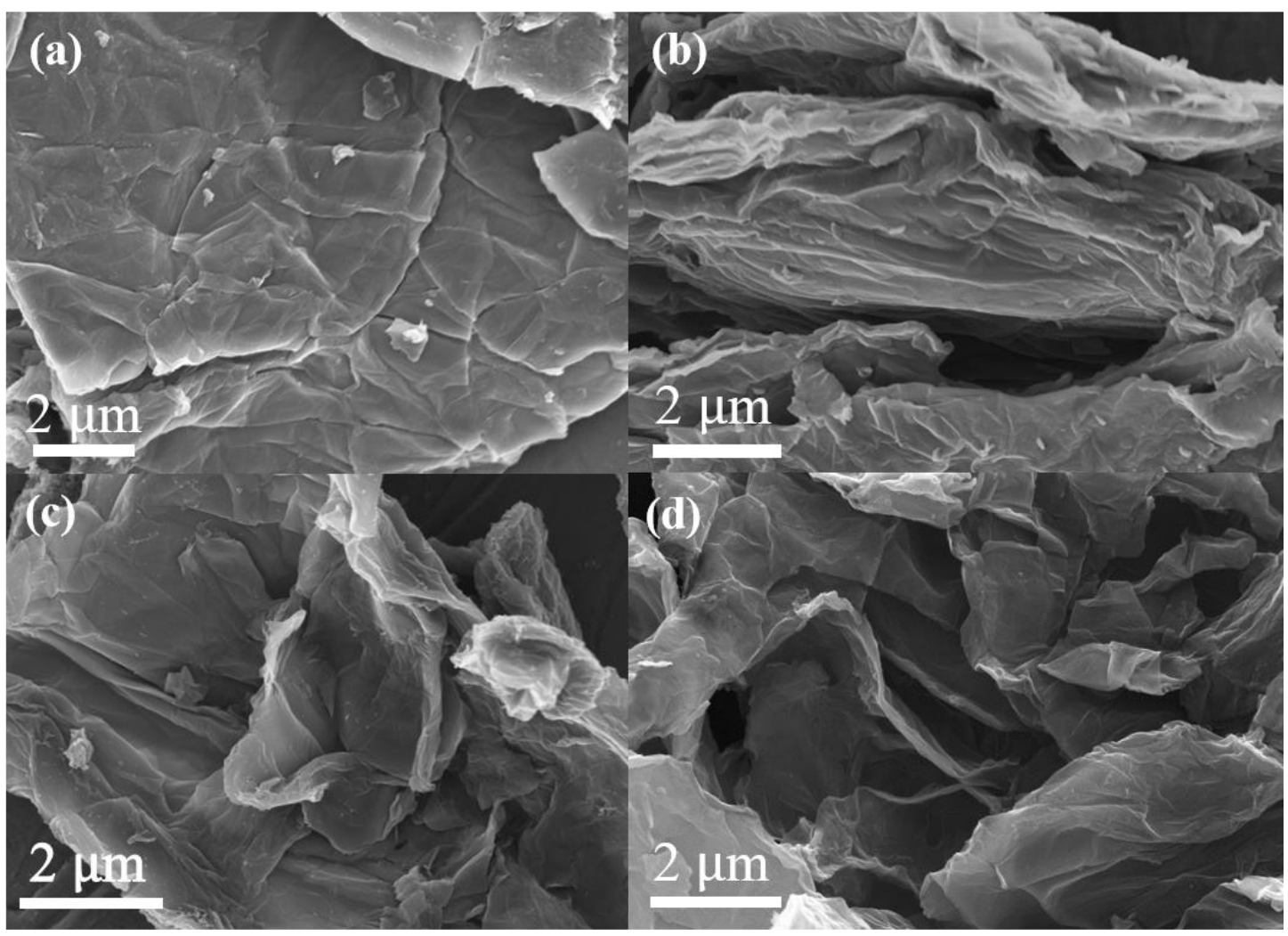

Fig. 1. SEM images of (a) GO, (b) rGO-air, (c) NG-Urea-air, and (d) N-rGO-air.

The SEM images in Fig. 1 and Fig. 2 display the morphologies of the nanocarbon catalysts.

Different from the smooth lamellar and severe stacked layers of GO (Figs. 1a and 2a), the obviously expanded and corrugated thin layers were spotted for rGO-air (Figs. $1 \mathrm{~b}$ and $2 \mathrm{~b}$ ). The $\mathrm{N}$-doped graphene presents an obviously curled structure as shown in Fig. 1c, which is further reflected in the TEM image of Fig. 2c in the crinkled sheets, due to the distortion of carbon 
lattice induced by the simultaneous reduction and $\mathrm{N}$-doping processes $[22,23]$. However, the partially aggregated structure was insignificant for $\mathrm{N}$-rGO-air with a veil-like sheet from $\mathrm{rGO}$ (Fig 1d), and the slightly curved structure at the boundaries of carbon network was also witnessed by the TEM image in Fig. $2 \mathrm{~d}$. Therefore, the carbon precursors may also influence the structure of the final product of graphene-based materials.

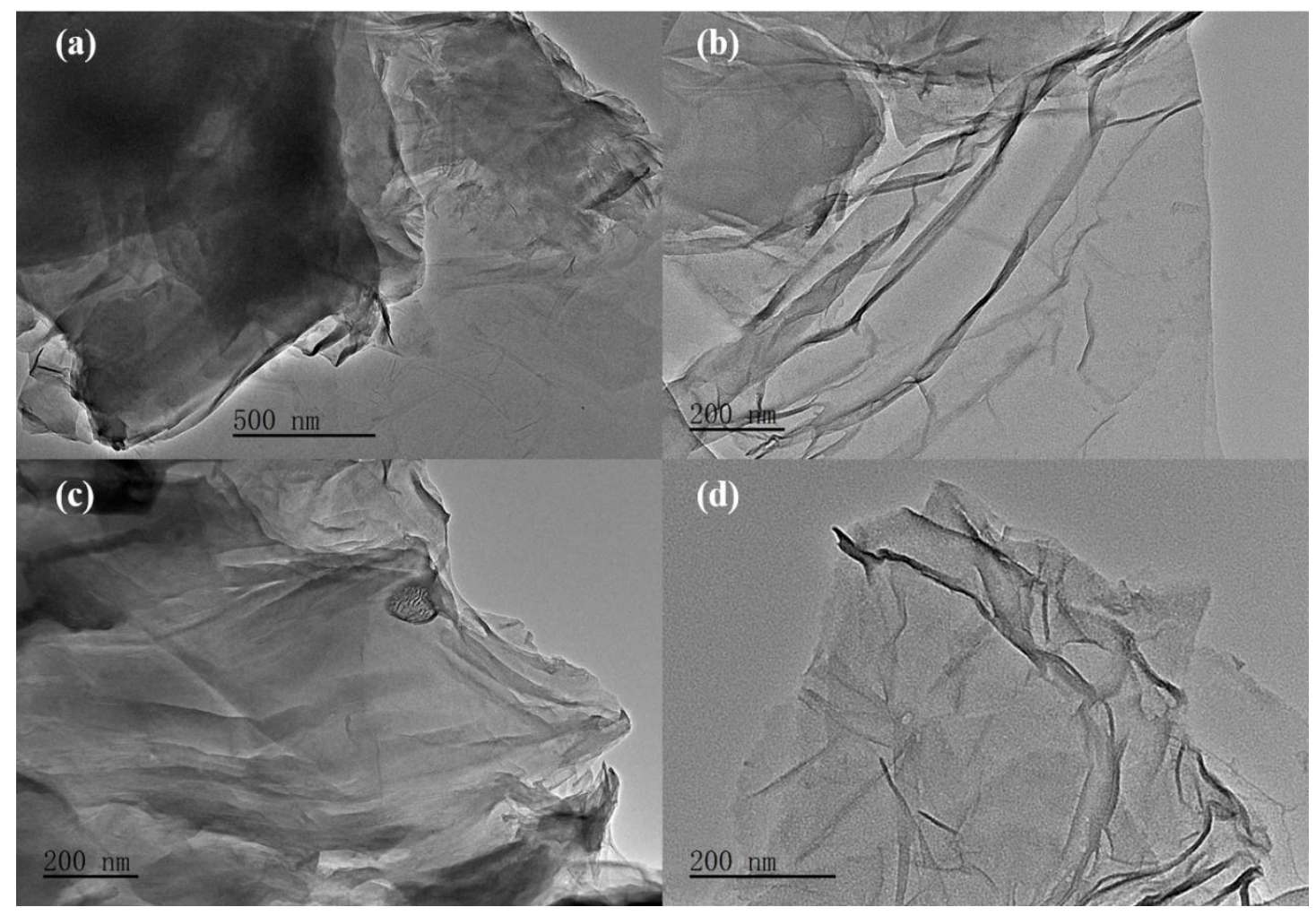

Fig. 2. TEM images of (a) GO, (b) rGO-air, (c) NG-Urea-air, and (d) N-rGO-air. 

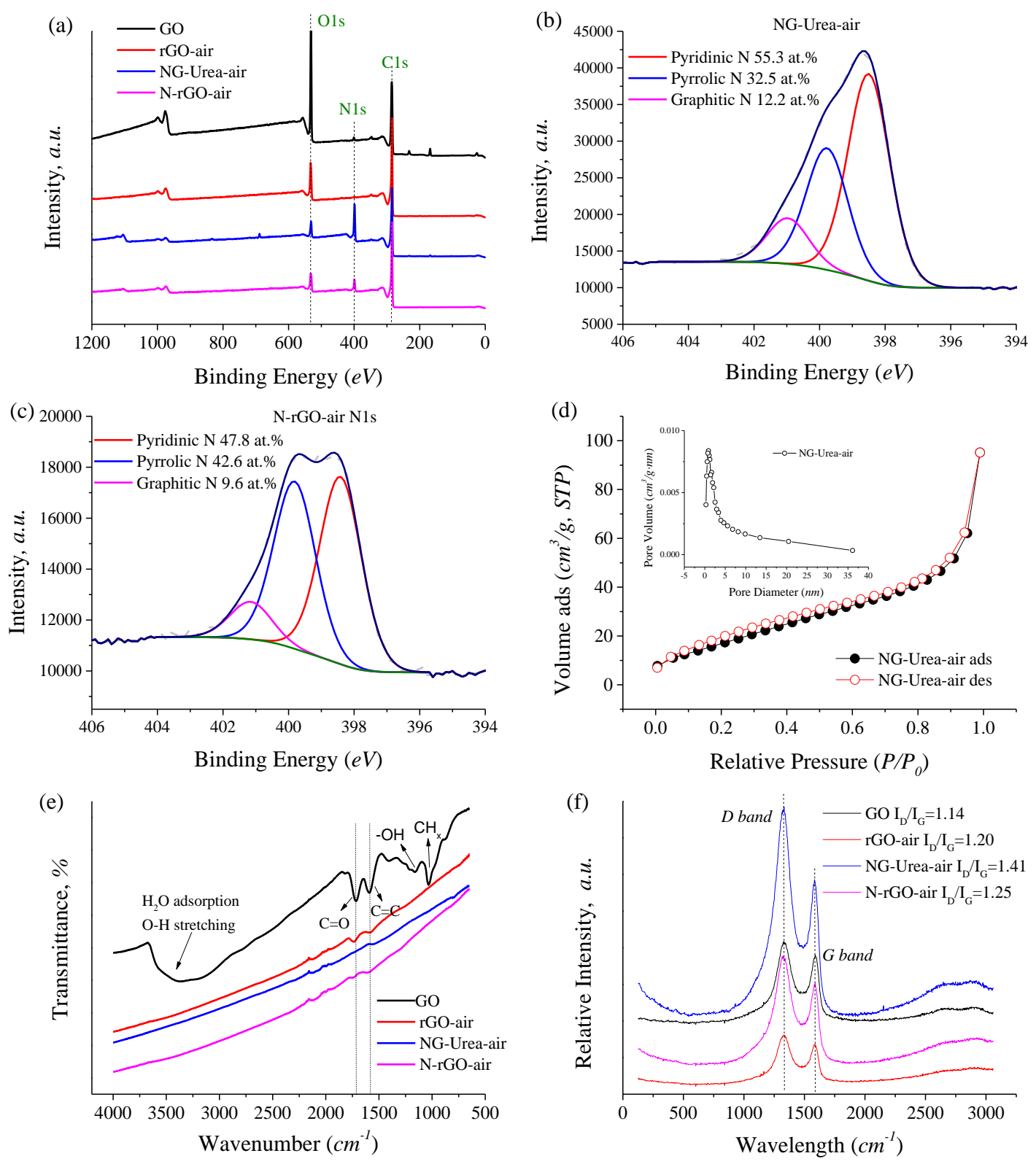

Fig. 3. (a) XPS surveys of nanocarbons, $N$ 1s high resolution spectra and fitting of (b) NGUrea-air and (c) N-rGO-air, (d) nitrogen sorption profile and pore size distribution of NG-Ureaair, (e) FTIR spectra and (g) Raman spectra of the carbocatalysts.

X-ray photoelectron spectroscopy (XPS) was utilized to analyze the chemical compositions of nanocarbon surface as shown in Figs. 3, S6 and S7. The XPS surveys in Fig. 3a illustrate that thermal annealing effectively eliminated the oxygen groups of the graphene oxide (31.4 at.\%, Table 1) to form a more reductive surface of rGO-air (14.3 at.\%). A small portion of nitrogen 
(1.2 at.\%) was induced in oxidation of graphite to synthesize graphene oxidation with strong oxidants $\left(\mathrm{KMnO}_{4}\right.$ and condensed $\left.\mathrm{HNO}_{3}\right)$, and the oxynitride $\left(\mathrm{GO}-\mathrm{C}-\mathrm{NO}_{2}\right)$ was decomposed as well during the thermal treatment. A lower oxygen contents were discovered for NG-Urea-air (4.9 at.\%), suggesting that the oxygen groups may play a crucial role in the binding with precursors and promote the $\mathrm{N}$-doping progress $[15,24]$. Besides, urea may work as the reducing agent during the thermal treatment to result in a better reducibility of rGO. The elemental analysis of XPS confirms that the nitrogen atoms have been successfully incorporated into the carbon framework, affording 18.7 at.\% and 7.3 at.\% nitrogen dopants for NG-Urea-air and NrGO-air, respectively. The $\mathrm{N}$ 1s fitting in Fig. 3b exhibits three main contributions located at 398.5, 399.8, and $401.0 \mathrm{eV}$ with respect to the pyridinic $\mathrm{N}$ (53.3 at.\%), pyrrolic $\mathrm{N}$ (32.5 at.\%), and graphitic N (12.2 at.\%) of NG-Urea-air accordingly. The three N-dopant species were also discovered in N-rGO-air as indicated in Fig. 3c. The proportion of graphitic N of NG-Urea-air and $\mathrm{N}$-rGO-air in total nitrogen was low due to the fact that the mild annealing temperature cannot produce high contents of substitutional N-doping into the carbon lattice. However, the moderate pyrolysis can achieve a very high doping level compared with graphene annealed at high temperatures (less than 10 at.\%), as the nitrogen dopants would decompose with the raising temperature and lead to a greater proportion of graphitic $\mathrm{N}$ [24, 25]. Hereby, the doping level and species can be tailored by adjusting the temperature and dosage of precursors. Fig. S8 manifested that the phenol oxidation rate was speeded up with the increased nitrogen amount in graphene, suggesting that the high doping level leads to a promoted catalytic performance for PMS activation. Our previous study also indicated raising the annealing temperature in inert atmosphere would result in a lower N-doping level due to the breakup of $\mathrm{C}-\mathrm{N}$ bond while affording a higher proportion of quaternary $\mathrm{N}$ benefiting from the better thermal stability of graphitic $\mathrm{N}$, which is simultaneously bonded with three carbon atoms with substitutional doping in the carbon framework [26]. 
Table 1: Physical and chemical properties of the nanocarbon catalysts.

\begin{tabular}{l|ccccc}
\hline & $\mathbf{C}$, at.\% & $\mathbf{N}$, at.\% & $\mathbf{O}$, at.\% & $\mathbf{I}_{\mathbf{D}} / \mathbf{I}_{\mathbf{G}}$ & $\mathbf{S S A}, \mathbf{~ m}^{2} / \mathbf{g}$ \\
\hline GO & 65.9 & 1.2 & 31.4 & 1.14 & 32.0 \\
rGO-air & 85.7 & - & 14.3 & 1.20 & 397.7 \\
NG-Urea-air & 76.4 & 18.7 & 4.9 & 1.41 & 71.4 \\
N-rGO-air & 84.0 & 7.3 & 8.7 & 1.33 & 362.0 \\
\hline
\end{tabular}

The nitrogen sorption isotherms in Fig. 3d indicate that the NG-Urea-air possesses a specific surface area (SSA) of $71.4 \mathrm{~m}^{2} / \mathrm{g}$, which is higher than GO $\left(32.0 \mathrm{~m}^{2} / \mathrm{g}\right.$, Fig. S9) yet much lower than $\mathrm{rGO}$-air of $397.7 \mathrm{~m}^{2} / \mathrm{g}$. The existence of organic matters during the annealing progress may restrict the thermal expansion of graphene oxide. Besides, the $\mathrm{N}$-doping process would produce the curvature structure of reduced GO as indicated in the SEM image, resulting in a decreased surface area [27]. However, N-rGO-air still achieved a high SSA $\left(362.0 \mathrm{~m}^{2} / \mathrm{g}\right)$, benefited from the precursor (rGO-air) with a porous structure and expanded layers. The FTIR spectra in Figs. 3e and S10 suggested that most of the oxygen-containing groups were removed after the heat treatment and N-doping process, which is consistent with the XPS studies. A weak and broad peak located at $1000-1300 \mathrm{~cm}^{-1}$ corresponding to $\mathrm{C}-\mathrm{O}(\mathrm{N})$ bond reported in previous studies was not discovered here, possibly due to the low-sensitivity of the instrument $[28,29]$. However, the XPS results revealed that the nitrogen dopants were successfully incorporated into the graphene framework and the EDS elemental mapping in Fig. S1 further confirmed that the nitrogen was uniformly distributed throughout the lattice of nitrogen doped graphene.

The Raman profiles in Figs. $3 \mathrm{f}$ and S11 indicate that all the nanocarbon catalysts exhibit two characteristic bands at 1331.8 ( $\mathrm{D}$ band) and $1583.2 \mathrm{~cm}^{-1}$ ( $\mathrm{G}$ band). The $\mathrm{D}$ band is associated with the defect numbers of graphene layer such as edges, non-six-atom rings, and heteroatom 
doping and functionalities, whereas the $G$ band is related to the $E_{2 g}$ mode vibration of $s^{2}$ hybridized carbon in the intact honeycomb unit [30, 31]. Therefore, the ratio of $\mathrm{D}$ band/G band intensities $\left(\mathrm{I}_{\mathrm{D}} / \mathrm{I}_{\mathrm{G}}\right)$ can reflect the defective degree of the carbon materials. The $\mathrm{I}_{\mathrm{D}} / \mathrm{I}_{\mathrm{G}}$ of $\mathrm{rGO}$-air was slightly higher than GO due to removal of oxygen moieties and releasing edging sites. It is well reported that the $\mathrm{N}$-doping can alter the spin culture and charge distribution of graphene then breaking the chemical inertness of graphene and promoting the catalytic performances in electrochemistry $[32,33]$. In this study, the $\mathrm{N}$-doping dramatically interrupted the carbon lattice and induced high defective degrees for NG-Urea-air (1.41) and N-rGO-air (1.32).

\subsection{Evaluation of catalytic oxidation over the carbocatalysts.}

The catalytic performances of the as-made nanocarbons were evaluated in activation of peroxymonosulfate for phenol oxidation. As indicated in Fig. 4a, PMS cannot oxidize phenol without activation and GO did not show any catalytic activity with only $8.8 \%$ phenol removal in 120 min. rGO-air presented a moderate oxidation efficiency with $42.6 \%$ phenol removal. Moreover, nitrogen-doped graphene demonstrated remarkably improvement with $100 \%$ phenol decomposition in less than 60 min. The reaction rates of GO, rGO-air, and NG-Urea-air were 0.0019, 0.0082, $0.094 \mathrm{~min}^{-1}$ (Fig. 4b), respectively, and N-doping induced 49.7- and 11.5-fold enhancement over GO and un-doped rGO-air. The NG-Urea-air also exhibited a superior activity over other nanocarbons such as $\mathrm{g}-\mathrm{C}_{3} \mathrm{~N}_{4}$, fullerene $\left(\mathrm{C}_{60}\right)$, commercial graphene nanosheets, and nanodiamond (Fig. S12). The NG-Urea-air attained 74.3\% total organic carbon (TOC) removal in $60 \mathrm{~min}$, and was also effective for oxidative removal of nitrobenzene, benzoic acid and 4-hydroxybenzoic acid (Fig. S13). The stability test was performed as shown in Fig. S14. Around $98.5 \%$ and $83.1 \%$ phenol decomposition rates were attained for the $2^{\text {nd }}$ and $3^{\text {rd }}$ runs accordingly, suggesting the excellent reusability of the carbocatlayst. The deactivation of the nitrogen-doped graphene might be ascribed to the alteration of surface chemistry and 
porous structure (Fig. S15). Many studies have reported that $\mathrm{N}$-doping can effectively break the inertness of $\mathrm{sp}^{2}$ hybridized carbon lattice and dramatically tailor the electron density and spin culture of the adjacent carbon, giving rise to the superb catalytic performances in electrochemistry, hydrocarbon conversion, and superoxide activation [34-36]. Our pioneering studies integrating with material design and theoretical calculations revealed that, due to a greater electronegativity $\left(\chi_{\mathrm{N}}=3.04\right)$ compared with the carbon atom $\left(\chi_{\mathrm{C}}=2.55\right), \mathrm{N}$-doping can effectivtly interrupt the highly-conjugated carbon network of graphene and induce the electron transport from the neighbouring carbon to nitrogen atoms, giving rise to positively charged carbon atoms $[37,38]$. These activated carbon atoms and domains in the carbon network demonstrated much a lower adsorption energy and a greater charge transfer tendency when bonding with PMS molecules and led to the promoted activity for metal-free oxidation. The experimental results indicated that the nitrogen-doped graphene with a higher proportion of graphitic $\mathrm{N}$ at a similar doping level exhibited a better catalytic activity for phenol oxidation, and the densities functional theory (DFT) calculations further evidenced that the adsorption of PMS molecules at the adjacent carbon of graphitic $\mathrm{N}$ exhibited the lowest adsorption energy and greatest tendency for electron transfer from carbon lattice to PMS for the activation of superoxide $\mathrm{O}-\mathrm{O}$ bond [26]. It is also supposed that the formation of a dipole moment in $\mathrm{N}$ doped graphene may act as a crucial step for the enhanced catalytic performances for PMS activation. 

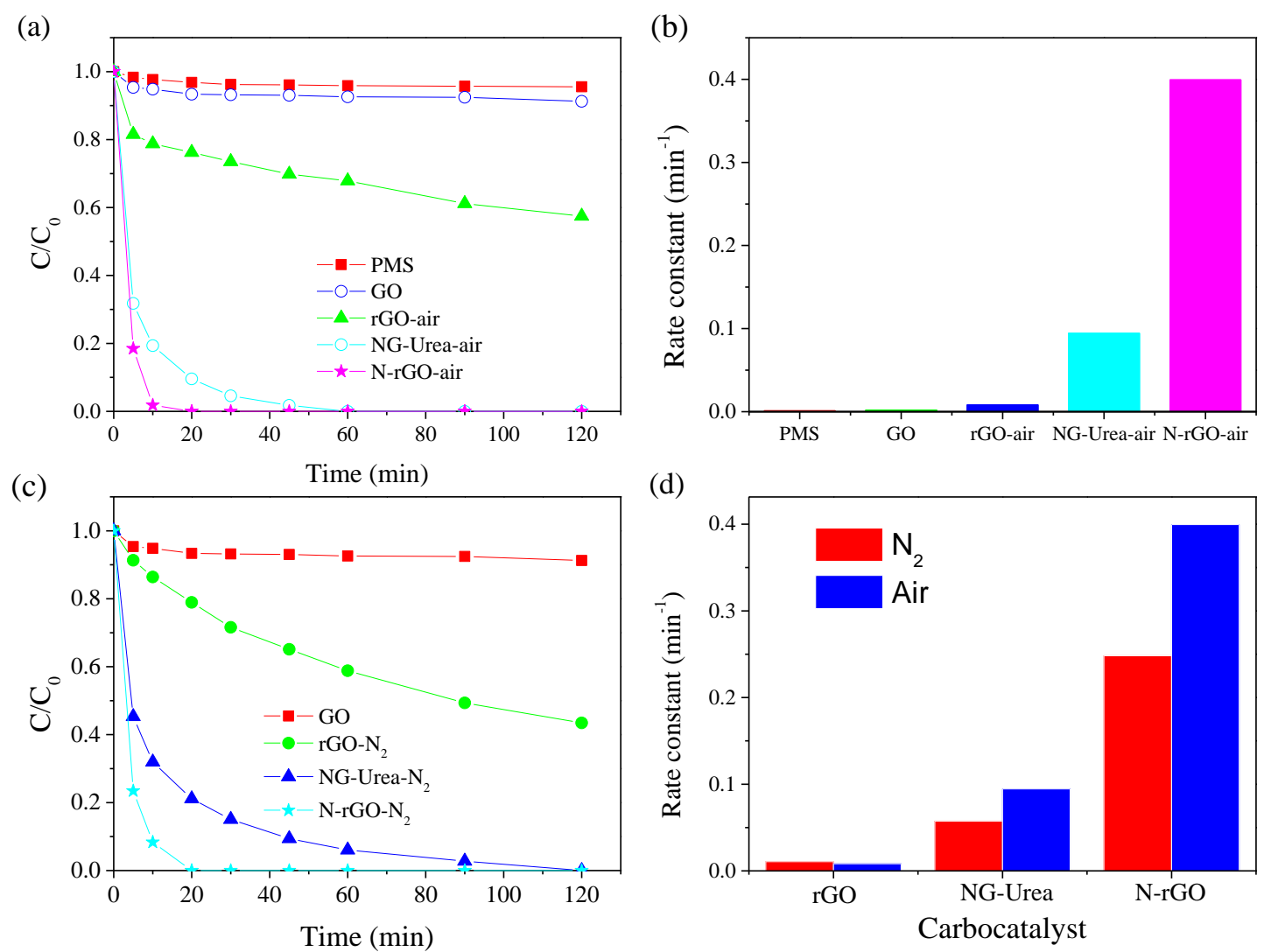

Fig. 4. (a) Catalytic activities of different nanocarbons for phenol oxidation, (b) pseudo-firstorder-based reaction rate constants of carbocatalysts, (c) phenol oxidation with nanocarbons synthesized in $\mathrm{N}_{2}$ atmosphere, and (d) comparison of pseudo-first-order reaction rate constants of carbocatalysts synthesized in different conditions.

Interestingly, N-rGO-air, synthesized by annealing rGO-air and urea, presented a higher catalytic activity for PMS activation than NG-Urea-air from GO. N-rGO-air gives a complete phenol oxidation in only 20 min with a high rate constant of $0.40 \mathrm{~min}^{-1}$ and harvests an Ndoping content of 7.3 at.\%. Several studies reported that the oxygen groups played a crucial role in the heteroatom doping processes $[24,39]$. The oxygen moieties would facilitate the adsorption and bonding with $\mathrm{N}$-precursors (e.g. $\mathrm{NH}_{3}$ ), and the decomposition of oxygen groups at high temperatures may leave certain 'active sites' which would react with the N-precursor to incorporate the nitrogen atoms into the carbon framework. With respect to $\mathrm{rGO}$, the re- 
constructing of graphene boundaries (edging sites) and lattice during annealing may also help attain certain amounts of $\mathrm{N}$-doping as shown in this study. Herein, the reduced graphene oxide, with a moderate oxygen doping level (14.3 at.\%) and high surface area $\left(397.7 \mathrm{~m}^{2} / \mathrm{g}\right)$, worked as a fantastic candidate for preparing $\mathrm{N}$-doped graphene. In spite of the relative lower $\mathrm{N}$-doped level (7.3 at.\%), N-rGO-air possesses a much higher specific surface area (SSA, $362.0 \mathrm{~m}^{2} / \mathrm{g}$ ) as shown in Table 1, which brings in exposing more active sites for PMS adsorption and phenol oxidation and exhibits a higher catalytic activity for PMS activation and phenol decomposition.

Additionally, the impact of the annealing atmosphere was investigated as indicated in Fig. 4. The carbocatalysts synthesized under the protection of $\mathrm{N}_{2}$ showed different activities compared with those under air. Fig. 4c displays that rGO gives a $66.5 \%$ phenol oxidation in $120 \mathrm{~min}$, and NG-Urea- $\mathrm{N}_{2}$ and N-rGO-N 2 achieved $100 \%$ phenol decomposition in 120 min and 20 min with the rate constants of $0.011,0.057$, and $0.25 \mathrm{~min}^{-1}$, respectively. Fig. $4 \mathrm{~d}$ suggests that the reaction rates of nitrogen doped graphene derived from air atmosphere are higher than the NGs under nitrogen. The reduction and doping procedures under air underwent mild combustion processes, leading to a higher defective degree (reflected by the higher $\mathrm{I}_{\mathrm{D}} / \mathrm{I}_{\mathrm{G}}$ values in Fig. $\mathrm{S} 11$ ) with more exposed edges and vacancies. Our recent study has revealed that the catalytic activity of graphene-based materials manifested a positively linear correlation between the defective degree and oxidative efficiency [40]. The theoretical studies also evidenced that zigzag and armchair edges with localized spins and unpaired $\pi$ electrons played a significant role in PMS adsorption, O-O bond activation, and electron transport for PMS activation [40]. Therefore, the rGO can be used as a better carbon precursor to prepare nitrogen-doped graphene with a desirable SSA, N-doping level, defective degree, and stunning catalytic activity. 

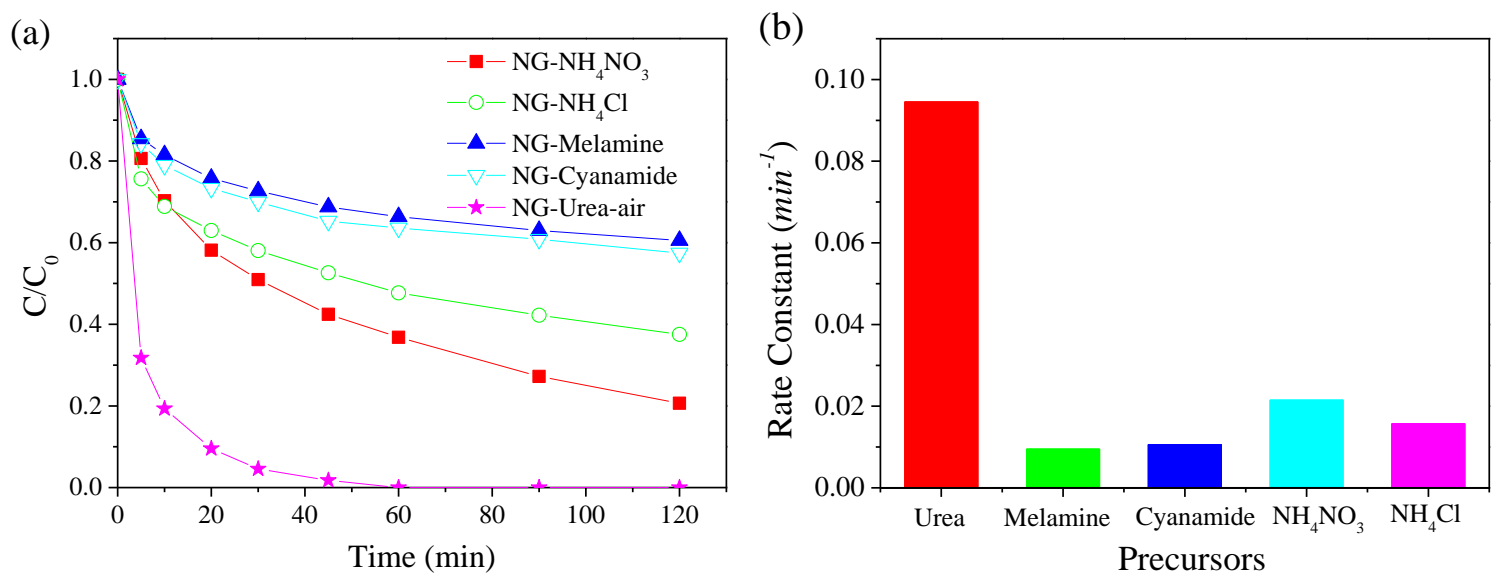

Fig. 5. (a) Phenol removal by nitrogen-doped graphene via different nitrogen precursors, (b) pseudo-first-order-based reaction rate constants of the nitrogen-doped graphene.

Table 2: Structure and surface chemistry of various N-doped graphene with different precursors.

\begin{tabular}{c|cccccccc}
\hline & $\begin{array}{c}\mathbf{C}, \\
\text { at.\% }\end{array}$ & $\begin{array}{c}\mathbf{N}, \\
\text { at.\% }\end{array}$ & $\begin{array}{c}\mathbf{O} \text { at.\% } \\
\text { at }\end{array}$ & $\begin{array}{c}\text { Pyridinic N, } \\
\text { at.\% }\end{array}$ & $\begin{array}{c}\text { Pyrrolic N, } \\
\text { at.\% }\end{array}$ & $\begin{array}{c}\text { Graphitic N, } \\
\text { at.\% }\end{array}$ & $\mathbf{I}_{\mathbf{D}} / \mathbf{I}_{\mathbf{G}}$ & $\begin{array}{c}\text { SSA, } \\
\mathbf{m}^{2} / \mathbf{g}\end{array}$ \\
\hline NG-Cyanamide & 55.7 & 35.7 & 8.6 & 54.7 & 31.8 & 13.6 & 1.21 & 49.6 \\
NG-Melamine & 48.5 & 42.1 & 9.4 & 48.7 & 39.5 & 11.8 & 1.19 & 63.1 \\
NG-Urea-air & 76.4 & 18.7 & 4.9 & 55.3 & 32.5 & 12.2 & 1.41 & 71.4 \\
NG-NH4 $_{4} \mathbf{C l}$ & 86.7 & 6.8 & 6.6 & 49.5 & 41.4 & 9.1 & 1.34 & 107.9 \\
NG-NH4NO $_{3}$ & 83.1 & 8.5 & 8.4 & 47.7 & 42.4 & 9.9 & 1.2 & 120.2 \\
\hline
\end{tabular}

Nevertheless many studies utilized various nitrogen-containing compounds as nitrogen sources to synthesize $\mathrm{N}$-doped graphene via thermal annealing or hydrothermal approaches, the influences of the precursors on $\mathrm{N}$-doping and catalytic performances of the products have not been systematically investigated yet. In this study, we utilized five different precursors stretching from the organic substances (e.g. urea, melamine, and cyanamide) to inorganic salts (ammonium nitrate and ammonium chloride) to probe the effects of precursors in $\mathrm{N}$-doped graphene on PMS activation. As shown in Fig. 5a, the NG-Melamine and NG-Cyanamide attained $42.6 \%$ and $39.5 \%$ phenol oxidation in $120 \mathrm{~min}$ with the rate constants of 0.0095 and $0.0106 \mathrm{~min}^{-1}$, respectively. $\mathrm{NG}-\mathrm{NH}_{4} \mathrm{NO}_{3}$ and $\mathrm{NG}-\mathrm{NH}_{4} \mathrm{Cl}$ achieved $79.4 \%$ and $62.5 \%$ organic 
removal and the rate constants are 0.0215 and $0.0157 \mathrm{~min}^{-1}$ accordingly. $\mathrm{N}$-doped graphene using urea as the precursor demonstrates the best activity among all the precursors. Despite the ultra-high nitrogen content of NG-Melamine (42.1 at.\%) and NG-Cyanamide (35.7 at.\%) as shown in Fig. S6 and Table S1, the FTIR spectra in Fig. S10 suggests that there existed condensed $\mathrm{C}_{\mathrm{x}} \mathrm{N}_{\mathrm{y}}$ substances due to the polymerization of the $\mathrm{N}$-rich organic precursors. The characteristic broad bands at $1250-1650 \mathrm{~cm}^{-1}$ can be ascribed to the stretching modes of lowmolecular-weight $\mathrm{CN}$ heterocycles and the sharp peaks at $740-830 \mathrm{~cm}^{-1}$ are attributed to the typical breathing mode of the tri-s-triazine units [41, 42]. This can be further confirmed by the unique pore size distribution and low SSAs as shown in Fig. S9, which is completely different from other nanocarbons in this study. The samples from melamine and cyanamide are apparent mixtures of $\mathrm{rGO}$ and condensed $\mathrm{C}_{\mathrm{x}} \mathrm{N}_{\mathrm{y}}$, and the nitrogen might not be completely incorporated into the carbon lattice, thus exhibiting the poor catalytic performances for phenol oxidation.

The NG- $\mathrm{NH}_{4} \mathrm{NO}_{3}$ and $\mathrm{NG}-\mathrm{NH}_{4} \mathrm{Cl}$ possessed lower doping levels with 8.5 at.\% and 6.8 at.\% nitrogen contents, yet the $\mathrm{N}$-doped graphene from ammonium salts exhibited better catalytic performances than NG-Melamine and NG-Cyanamide (Fig. S6 and Table 2). The ammonium salts would decompose during the thermal annealing and release $\mathrm{NH}_{3}$ (the doping agent) and other gasses $\left(\mathrm{N}_{2}, \mathrm{~N}_{2} \mathrm{O}\right.$, or $\left.\mathrm{HCl}\right)$, which facilitate the formation of porous $\mathrm{N}$-doped graphene with larger SSAs, pore size, and pore volume (Fig. S9 and Table S2). Nevertheless, the Ndoped graphene from urea presented a smaller specific surface area compared with NG$\mathrm{NH}_{4} \mathrm{NO}_{3}$ and $\mathrm{NG}-\mathrm{NH}_{4} \mathrm{Cl}$, NG-Urea-air contains a higher $\mathrm{N}$-doped level (18.7 at.\%) and the lowest oxygen level (4.9 at.\%) among the NGs and demonstrated the best activity for catalytic oxidation. The thermal decomposition of urea can produce $\mathrm{NH}_{3}$ and $\mathrm{CO}_{2}$, which simultaneously enabled the $\mathrm{N}$-doping, tailored the surface chemistry and pore structure, and modified the acidity/alkalinity of graphene basal plane. The annealing process with a slow 
heating rate $\left(5^{\circ} \mathrm{C} / \mathrm{min}\right)$ also effectively inhibited the polycondensation of urea as the process only occurs during the rapidly thermal polymerization in the presence of large amounts of urea. Therefore, the urea achieved a high doping level in nitrogen doped graphene meanwhile attaining a better reducibility of the carbon lattice, giving rise to more active sites for PMS activation and improved electron-transport capability of the graphene network. The N-rGO-air presents a similar $\mathrm{N}$-doping level to $\mathrm{NG}-\mathrm{NH}_{4} \mathrm{Cl}$ and $\mathrm{NG}-\mathrm{NH}_{4} \mathrm{NO}_{3}$ and a much higher surface area $\left(362.0 \mathrm{~m}^{2} / \mathrm{g}\right)$, which is almost 3.3 times of $\mathrm{NG}-\mathrm{NH}_{4} \mathrm{Cl}\left(107.9 \mathrm{~m}^{2} / \mathrm{g}\right)$ and 3.0 times of NG$\mathrm{NH}_{4} \mathrm{NO}_{3}\left(120.2 \mathrm{~m}^{2} / \mathrm{g}\right)$. The high surface area enables the N-rGO-air with more active centres to be exposed to PMS molecules, giving rise to a better catalytic activity.

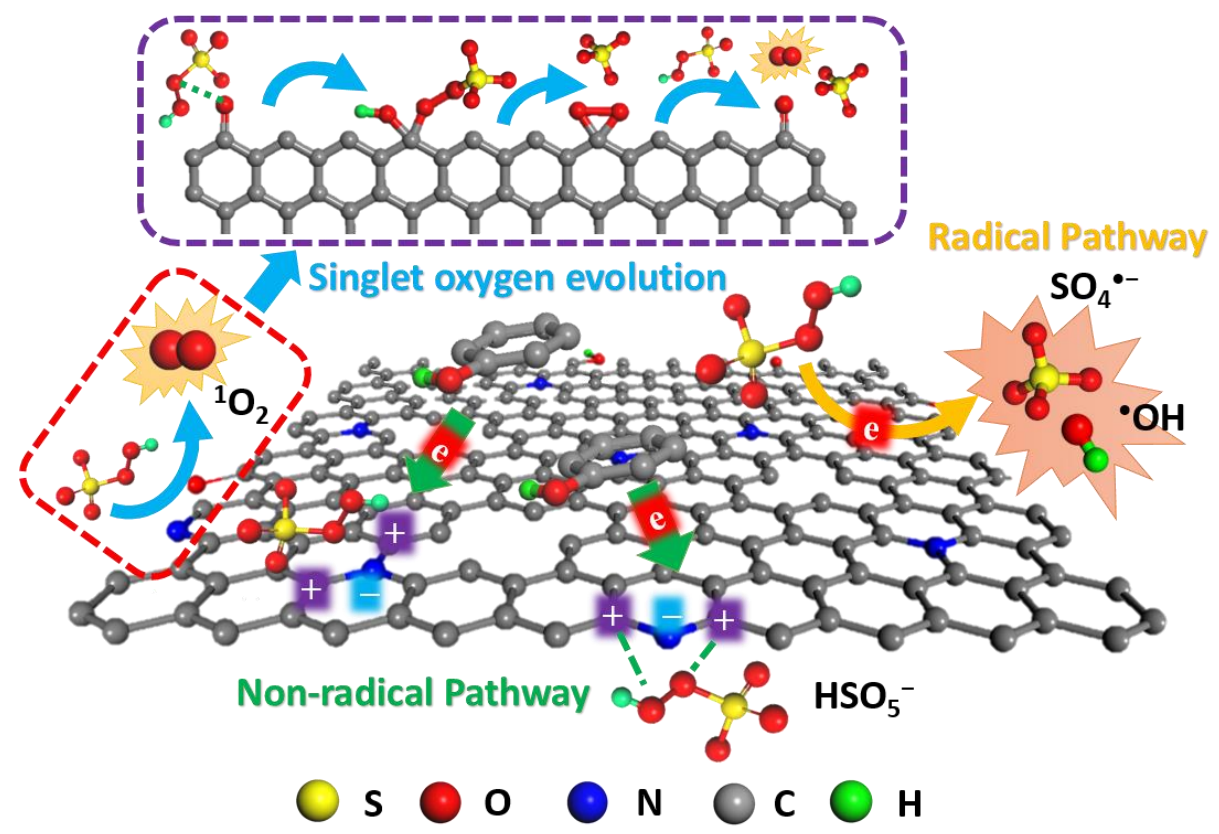

Fig. 6. Mechanism of PMS activation on N-doped graphene for phenol oxidation.

\subsection{Mechanism of PMS activation}

In a preliminary study, we discovered that $\mathrm{N}$-doping into the highly graphitic single-walled carbon nanotubes (N-SWCNT) would mediate a nonradical oxidative pathway, in which the organics was oxidized directly on the carbon surface without generation of free radicals [43]. Both the radical and non-radical mechanisms contribute to the complete oxidation of 
contaminants in the novel metal-free system. A similar process was also discovered in a peroxydisulfate $(\mathrm{PDS}) / \mathrm{CuO}$ system in which the persulfate was activated via out-shell interaction with $\mathrm{CuO}$, and subsequently oxidized the target organic through electron extraction [44]. Lee and co-workers also revealed that the efficient CNT/PDS system is not dependent on the generation of sulfate radicals for organic oxidation [45]. In this study, we applied high dosage of alcohols into the oxidative system. Both methanol $(\mathrm{MeOH})$ and ethanol $(\mathrm{EtOH})$ can rapidly quench hydroxyl radicals $\left({ }^{\circ} \mathrm{OH}\right)$ and sulfate radicals $\left(\mathrm{SO}_{4}{ }^{--}\right)$in aqueous solution with a high reaction rate (shown in Table S3). However, a high ratio of the radical scavengers $\left(\mathrm{M}_{\mathrm{MeOH}}\right.$ : $\left.\mathrm{M}_{\mathrm{PMS}}=1000\right)$ did not completely terminate the oxidative reactions, and an excellent phenol oxidation efficiency was still achieved with complete organics removal in $120 \mathrm{~min}$, which is intrinsically different from the classical radical-based $\mathrm{Co}_{3} \mathrm{O}_{4} / \mathrm{PMS}$ system in which the reaction was dramatically shut down by the alcohols (Fig. S16). The outstanding efficiency of PMS activation on nitrogen-doped graphene is also contributed by the non-radical process, in which the PMS molecules are activated at N-doped domains and followed by readily oxidation of target organics via electron transfer as illustrated in Fig. 6. Besides, the undoped $\mathrm{sp}^{2}$ hybridized carbon network with abundant free-flowing electrons and edging sites with unpaired electrons are able to be transferred to PMS to form small quantity of free radicals $\left(\mathrm{SO}_{4}{ }^{--}\right.$and $\left.{ }^{\circ} \mathrm{OH}\right)$ as the alcohols indeed slow down the oxidation.

(a)

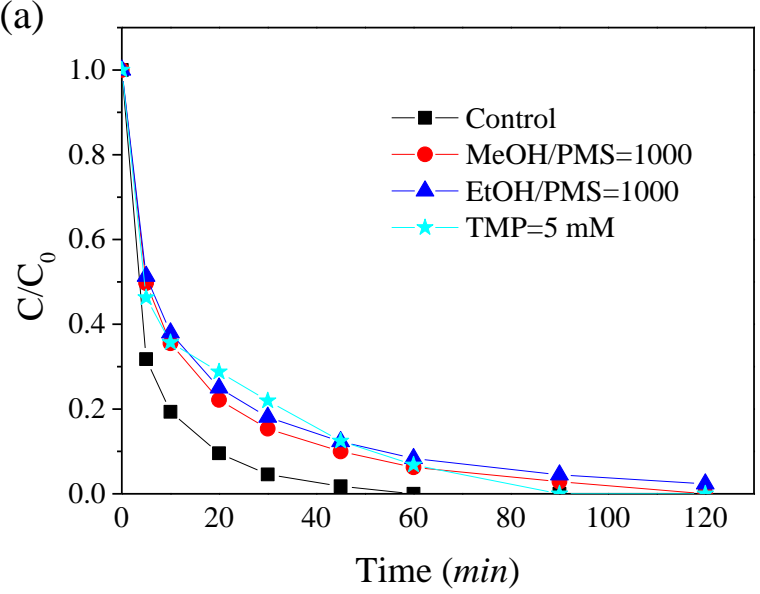

(b)

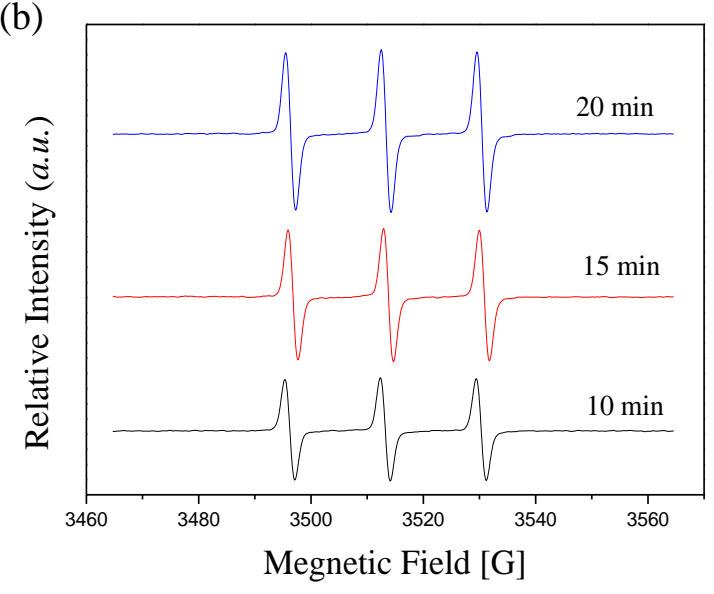


Fig. 7. (a) The effects of radical quenching agents on phenol oxidation in NG-Urea-air/PMS, (b) EPR spectra of captured signals of singlet oxygen.

More recently, Zhou et al. reported that benzoquinone can activate PMS to generate singlet oxygen $\left({ }^{1} \mathrm{O}_{2}\right)$ for the oxidation of sulfamethoxazone via a nonradical process [46]. The carbonyl group $(\mathrm{C}=\mathrm{O})$ plays a vital role in interacting with PMS through nucleophilic addition and mediating a peroxide intermediate for the revolution of singlet oxygen. As graphene-based materials derived from GO also contains certain amounts of ketonic groups, which may induce singlet oxygen in this system. Sodium azide $\left(\mathrm{NaN}_{3}\right)$ was able to eliminate the singlet oxygen with a high reaction rate (Table S3). As shown in Fig. 7a, the time for complete phenol degradation increased from 60 to 90 min when $5 \mathrm{mM} \mathrm{NaN}_{3}$ was introduced into the oxidative system, suggesting that the singlet oxygen was generated and partially contributed to the organics removal. This is further supported by the in situ EPR spectra that singlet oxygen signals $\left(\alpha_{\mathrm{N}}=16.9 \mathrm{G}, \mathrm{g}=0.0054\right)$ were captured with increased intensities as the reaction progress (Fig. 7b). The ketonic groups $(\mathrm{C}=\mathrm{O})$ at the boundaries of $\mathrm{sp}^{2}$-conjugated carbon lattice could be the active sites for the evolution of singlet oxygen as elucinated in Fig. 6. As a result, $\mathrm{N}$-doped graphene/PMS is a complicated system involving multi-oxidative pathways, which is intrinsically different from the radical-dominated metal-based AOPs systems.

\subsection{Effects of solution $\mathrm{pH}$ and halogen ions}

The impact of chloride ions was studied in the carbon-catalyzed oxidative system. Generally, chloride ions would slow down the oxidation by competitive reactions with sulfate radicals and hydroxyl radicals to produce chloride radicals $\left(\mathrm{Cl}^{\circ}\right)$ which possess a lower redox potential. Fig. 8a shows that the reaction rate was slightly decreased with an addition of $2 \mathrm{mM} \mathrm{Cl}^{-}$, however, the reaction was promoted when excessive chloride ions of $5 \mathrm{mM}$ and $10 \mathrm{mM}$ were introduced with complete phenol removal in 30 and $5 \mathrm{~min}$, respectively. The chloride ions not only 
scavenge the reactive radicals which partially contribute to the oxidation, but also compete with the organics to donate an electron to form $\mathrm{Cl}^{\circ}$ via the nonradical process $[47,48]$. The minor chloride ions $(2 \mathrm{mM})$ would scavenge the sulfate radicals and compete with the target organics via the nonradical oxidation to form $\mathrm{Cl}^{\circ}$ with a relative low redox capability, leading to a decreased performance for organic degradation. When excessive chloride ions (5 and $10 \mathrm{mM}$ ) were introduced into the system, the produced $\mathrm{Cl}^{\circ}$ would then quickly react with superabundant $\mathrm{Cl}^{-}$to form chlorine radicals $\left(\mathrm{Cl}_{2}{ }^{\circ-}, 2.1 \mathrm{~V}\right)$ with a higher oxidative potential, which tend to attack the electron-rich organics and induce the enhanced efficiency of phenol removal [49].

(a)

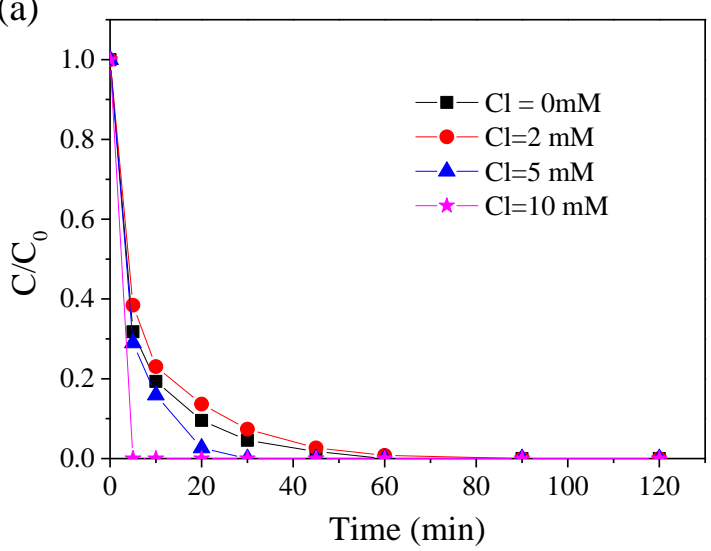

(b)

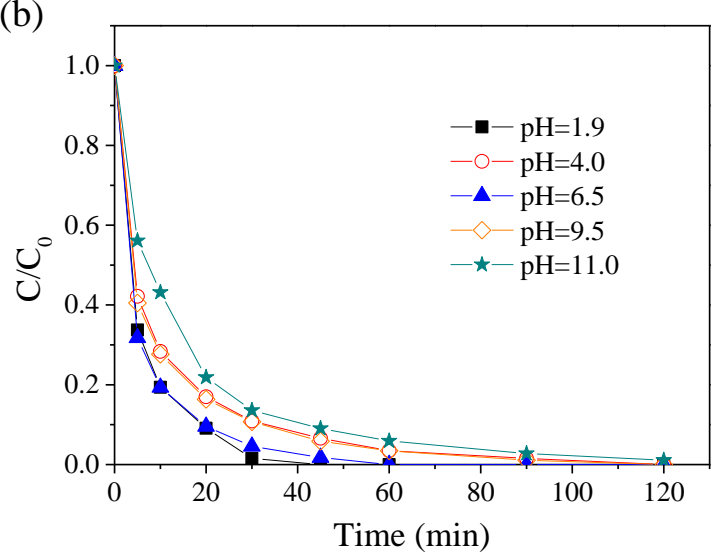

Fig. 8. The effects of (a) chloride ions, and (b) initial $\mathrm{pH}$ values on organics removal by PMS activation on NG-Urea-air.

The influence of initial solution pH was also investigated in PMS activation with NG-Urea-air. The $\mathrm{pH}$ has been reported to dramatically affect the catalytic efficiency of heterogeneously metal catalysts for PMS activation [50, 51]. The acidic environment influences the dissociation of PMS $\left(\mathrm{HSO}_{5}{ }^{-}, \mathrm{pK}_{\mathrm{a} 1}<0, \mathrm{pK}_{\mathrm{a} 2}=9.88\right)$, hereby impacting the ionic existing forms and reactivity of PMS molecules. Additionally, the acidic/basic condition of the reaction solutions can impact the catalytic performance of metal catalysts as the strong acidic condition can destroy the metal crystalline structure and lead to severe metal leaching. In this study, Fig. 8b shows that the complete phenol degradation was achieved at the pHs of 1.9, 4.0, 6.5, 9.5, and 
11.0 within $120 \mathrm{~min}$. It is discovered that the reaction rate at $\mathrm{pH} 4.0$ is lower than that $\mathrm{pH}$ at 6.5, because at lower $\mathrm{pH}$, the PMS was presented as its mother acid $\mathrm{H}_{2} \mathrm{SO}_{5}$, herein it is more difficult to be activated by carbocatalysts than its neutral $\left(\mathrm{HSO}_{5}^{-}\right)$and basic format $\left(\mathrm{HSO}_{5}{ }^{2-}\right)$ The strong basic condition will possibly functionalize the carbon surface with hydroxyl ions, resulting in the electrostatic repulsive forces between the PMS and the active sites and an inferior activity for PMS adsorption and catalytic oxidation (similar to metal oxides), despite the fact that a base is reported to be able to activate PMS [52]. Herein, the reaction rate underwent a slight decline at higher $\mathrm{pH}$ of 9.5 . The nanocarbons still adapt to a wide $\mathrm{pH}$ range for organic oxidation without inducing toxic metal leaching compared with metal catalysts, exhibiting the superb merits of carbocatalysis for environmental remediation in complicated scenarios.

\section{Conclusions}

In summary, nitrogen-doped graphene was synthesized via a facile thermal combustion approach and applied for heterogeneous activation of peroxymonosulfate for organic oxidation. The N-doped graphene derived from urea demonstrated a significant enhancement toward catalytic decomposition of toxic contaminants. The influences of precursors and doping conditions were investigated. Reduced graphene oxide with a large specific area and a proper amount of oxygen groups could be utilized as a potential candidate for $\mathrm{N}$-doping with a larger SSA and considerable doping level, showing much better activity than those from graphene oxide. The mild annealing and doping processes favored the oxidative atmosphere due to the induced structure defects such as edging sites and vacancies which enabled carbon reconstruction for $\mathrm{N}$-doping. Both organic substances and inorganic salts were applied as the nitrogen precursors for nitrogen doping and urea was discovered to be the best precursor with a high doping level and better reducibility without any polycondensation. The high phenol 
removal efficiency with carbocatalysis was benefited from the nonradical oxidation meanwhile involving singlet oxygen, and a few other free radicals. The graphene-based system achieved a high organic removal rate at various $\mathrm{pH}$ values and in the presence of chloride ions. This study provides a fundamental understanding of the nitrogen doping progress in carbon framework as well as the mechanistic insights into peroxymonosulfate activation by nitrogendoped graphene. The results would contribute to designing novel carbon materials with efficient strategy of heteroatom doping and revealing the meal-free carbocatalysis in environmental science.

\section{Acknowledgement}

The authors acknowledge the financial supports from Australian Research Council (ARC DP150103026).

\section{Appendix A. Supplementary Data}

Supplementary data, EDS elemental mapping, XPS spectra, nitrogen sorption profiles, Raman spectra, FTIR spectra, and associated experimental tests, related with this article can be found online, at http://********.

\section{References}

[1] A.K. Geim, Graphene: Status and prospects, Science 324 (2009) 1530-1534.

[2] A.K. Geim, K.S. Novoselov, The rise of graphene, Nat. Mater. 6 (2007) 183-191.

[3] Z.P. Chen, W.C. Ren, L.B. Gao, B.L. Liu, S.F. Pei, H.M. Cheng, Three-dimensional flexible and conductive interconnected graphene networks grown by chemical vapour deposition, Nat. Mater. 10 (2011) 424-428.

[4] C. Berger, Z.M. Song, X.B. Li, X.S. Wu, N. Brown, C. Naud, D. Mayou, T.B. Li, J. Hass, A.N. Marchenkov, E.H. Conrad, P.N. First, W.A. de Heer, Electronic confinement and coherence in patterned epitaxial graphene, Science 312 (2006) 1191-1196.

[5] M. Lotya, Y. Hernandez, P.J. King, R.J. Smith, V. Nicolosi, L.S. Karlsson, F.M. Blighe, S. De, Z.M. Wang, I.T. McGovern, G.S. Duesberg, J.N. Coleman, Liquid phase production of 
graphene by exfoliation of graphite in surfactant/water solutions, J. Am. Chem. Soc. 131 (2009) 3611-3620.

[6] K.S. Novoselov, A.K. Geim, S.V. Morozov, D. Jiang, Y. Zhang, S.V. Dubonos, I.V. Grigorieva, A.A. Firsov, Electric field effect in atomically thin carbon films, Science 306 (2004) 666-669.

[7] K. Parvez, Z.S. Wu, R.J. Li, X.J. Liu, R. Graf, X.L. Feng, K. Mullen, Exfoliation of graphite into graphene in aqueous solutions of inorganic salts, J. Am. Chem. Soc. 136 (2014) 60836091.

[8] S. Park, R.S. Ruoff, Chemical methods for the production of graphenes, Nat. Nanotechnol. 4 (2009) 217-224.

[9] W.S. Hummers, R.E. Offeman, Preparation of graphitic oxide, J. Am. Chem. Soc. 80 (1958) 1339-1339.

[10] Y.W. Zhu, M.D. Stoller, W.W. Cai, A. Velamakanni, R.D. Piner, D. Chen, R.S. Ruoff, Exfoliation of graphite oxide in propylene carbonate and thermal reduction of the resulting graphene oxide platelets, ACS Nano 4 (2010) 1227-1233.

[11] S. Stankovich, D.A. Dikin, R.D. Piner, K.A. Kohlhaas, A. Kleinhammes, Y. Jia, Y. Wu, S.T. Nguyen, R.S. Ruoff, Synthesis of graphene-based nanosheets via chemical reduction of exfoliated graphite oxide, Carbon 45 (2007) 1558-1565.

[12] M.J. Fernandez-Merino, L. Guardia, J.I. Paredes, S. Villar-Rodil, P. Solis-Fernandez, A. Martinez-Alonso, J.M.D. Tascon, Vitamin C is an ideal substitute for hydrazine in the reduction of graphene oxide suspensions, J. Phys. Chem. C. 114 (2010) 6426-6432.

[13] X.K. Kong, C.L. Chen, Q.W. Chen, Doped graphene for metal-free catalysis, Chem. Soc. Rev. 43 (2014) 2841-2857.

[14] H.T. Liu, Y.Q. Liu, D.B. Zhu, Chemical doping of graphene, J. Mater. Chem. 21 (2011) 3335-3345.

[15] Z.H. Sheng, L. Shao, J.J. Chen, W.J. Bao, F.B. Wang, X.H. Xia, Catalyst-free synthesis of nitrogen-doped graphene via thermal annealing graphite oxide with melamine and its excellent electrocatalysis, ACS Nano 5 (2011) 4350-4358.

[16] Z.Y. Lin, G. Waller, Y. Liu, M.L. Liu, C.P. Wong, Facile synthesis of nitrogen-doped graphene via pyrolysis of graphene oxide and urea, and its electrocatalytic activity toward the oxygen-reduction reaction, Adv. Energy. Mater. 2 (2012) 884-888.

[17] Y. Zheng, Y. Jiao, L. Ge, M. Jaroniec, S.Z. Qiao, Two-step boron and nitrogen doping in graphene for enhanced synergistic catalysis, Angew. Chem. Int. Ed. 52 (2013) 3110-3116. 
[18] H.Q. Sun, S.Z. Liu, G.L. Zhou, H.M. Ang, M.O. Tade, S.B. Wang, Reduced graphene oxide for catalytic oxidation of aqueous organic pollutants, ACS Appl. Mater. Interfaces 4 (2012) 5466-5471.

[19] X.G. Duan, Z.M. Ao, H.Q. Sun, L. Zhou, G.X. Wang, S.B. Wang, Insights into N-doping in single-walled carbon nanotubes for enhanced activation of superoxides: a mechanistic study, Chem. Commun. 51 (2015) 15249-15252.

[20] J.C. Espinosa, S. Navalon, A. Primo, M. Moral, J.F. Sanz, M. Alvaro, H. Garcia, Graphenes as efficient metal-free fenton catalysts, Chem. Eur. J. 21 (2015) 11966-11971.

[21] Y.X. Wang, Y.B. Xie, H.Q. Sun, J.D. Xiao, H.B. Cao, S.B. Wang, Efficient catalytic ozonation over reduced graphene oxide for p-hydroxylbenzoic acid (PHBA) destruction: Active site and mechanism, ACS Appl. Mater. Interfaces 8 (2016) 9710-9720.

[22] Y.Z. Su, Y. Zhang, X.D. Zhuang, S. Li, D.Q. Wu, F. Zhang, X.L. Feng, Low-temperature synthesis of nitrogen/sulfur co-doped three-dimensional graphene frameworks as efficient metal-free electrocatalyst for oxygen reduction reaction, Carbon 62 (2013) 296-301.

[23] Y.Y. Shao, S. Zhang, M.H. Engelhard, G.S. Li, G.C. Shao, Y. Wang, J. Liu, I.A. Aksay, Y.H. Lin, Nitrogen-doped graphene and its electrochemical applications, J. Mater. Chem. 20 (2010) 7491-7496.

[24] X.L. Li, H.L. Wang, J.T. Robinson, H. Sanchez, G. Diankov, H.J. Dai, Simultaneous nitrogen doping and reduction of graphene oxide, J. Am. Chem. Soc. 131 (2009) 15939-15944. [25] D.H. Deng, X.L. Pan, L.A. Yu, Y. Cui, Y.P. Jiang, J. Qi, W.X. Li, Q.A. Fu, X.C. Ma, Q.K. Xue, G.Q. Sun, X.H. Bao, Toward N-doped graphene via solvothermal synthesis, Chem. Mater. 23 (2011) 1188-1193.

[26] X.G. Duan, Z.M. Ao, H.Q. Sun, S. Indrawirawan, Y.X. Wang, J. Kang, F.L. Liang, Z.H. Zhu, S.B. Wang, Nitrogen-doped graphene for generation and evolution of reactive radicals by metal-free catalysis, ACS Appl. Mater. Interfaces 7 (2015) 4169-4178.

[27] H.Q. Sun, Y.X. Wang, S.Z. Liu, L. Ge, L. Wang, Z.H. Zhu, S.B. Wang, Facile synthesis of nitrogen doped reduced graphene oxide as a superior metal-free catalyst for oxidation, Chem. Commun. 49 (2013) 9914-9916.

[28] S. Chen, J.J. Duan, M. Jaroniec, S.Z. Qiao, Nitrogen and oxygen dual-doped carbon hydrogel film as a substrate-free electrode for highly efficient oxygen evolution reaction, Adv. Mater. 26 (2014) 2925-2930.

[29] S. Chen, J.J. Duan, Y.H. Tang, B. Jin, S.Z. Qiao, Molybdenum sulfide clusters-nitrogendoped graphene hybrid hydrogel film as an efficient three-dimensional hydrogen evolution electrocatalyst, Nano Energy 11 (2015) 11-18. 
[30] A.C. Ferrari, J.C. Meyer, V. Scardaci, C. Casiraghi, M. Lazzeri, F. Mauri, S. Piscanec, D. Jiang, K.S. Novoselov, S. Roth, A.K. Geim, Raman spectrum of graphene and graphene layers, Phys. Rev. Lett. 97 (2006) 187401.

[31] S. Berciaud, S. Ryu, L.E. Brus, T.F. Heinz, Probing the intrinsic properties of exfoliated graphene: Raman spectroscopy of free-standing monolayers, Nano Lett. 9 (2009) 346-352.

[32] J. Liang, Y. Jiao, M. Jaroniec, S.Z. Qiao, Sulfur and nitrogen dual-doped mesoporous graphene electrocatalyst for oxygen reduction with synergistically enhanced performance, Angew. Chem. Int. Ed. 51 (2012) 11496-11500.

[33] L.F. Lai, J.R. Potts, D. Zhan, L. Wang, C.K. Poh, C.H. Tang, H. Gong, Z.X. Shen, L.Y. Jianyi, R.S. Ruoff, Exploration of the active center structure of nitrogen-doped graphene-based catalysts for oxygen reduction reaction, Energ. Environ. Sci. 5 (2012) 7936-7942.

[34] S.Y. Wang, D.S. Yu, L.M. Dai, Polyelectrolyte functionalized carbon nanotubes as efficient metal-free electrocatalysts for oxygen reduction, J. Am. Chem. Soc. 133 (2011) 51825185.

[35] J.L. Long, X.Q. Xie, J. Xu, Q. Gu, L.M. Chen, X.X. Wang, Nitrogen-doped graphene nanosheets as metal-free catalysts for aerobic selective oxidation of benzylic alcohols, ACS Catal. 2 (2012) 622-631.

[36] S. Indrawirawan, H.Q. Sun, X.G. Duan, S.B. Wang, Low temperature combustion synthesis of nitrogen-doped graphene for metal-free catalytic oxidation, J. Mater. Chem. A 3 (2015) 3432-3440.

[37] X.G. Duan, K. O'Donnell, H.Q. Sun, Y.X. Wang, S.B. Wang, Sulfur and nitrogen codoped graphene for metal-free catalytic oxidation reactions, Small 11 (2015) 3036-3044.

[38] X.G. Duan, Z.M. Ao, D.G. Li, H.Q. Sun, L. Zhou, A. Suvorova, M. Saunders, G.X. Wang, S.B. Wang, Surface-tailored nanodiamonds as excellent metal-free catalysts for organic oxidation, Carbon 103 (2016) 404-411.

[39] Y.M. Lin, D.S. Su, Fabrication of nitrogen-modified annealed nanodiamond with improved catalytic activity, ACS Nano 8 (2014) 7823-7833.

[40] X.G. Duan, H.Q. Sun, Z.M. Ao, L. Zhou, G.X. Wang, S.B. Wang, Unveiling the active sites of graphene-catalyzed peroxymonosulfate activation, Carbon 107 (2016) 371-378.

[41] T.Y. Ma, Y.H. Tang, S. Dai, S.Z. Qiao, Proton-functionalized two-dimensional graphitic carbon nitride nanosheet: An excellent metal-free biosensing platform, Small 10 (2014) 23822389 . 
[42] G.Z. Liao, S. Chen, X. Quan, H.T. Yu, H.M. Zhao, Graphene oxide modified $\mathrm{g}_{-} \mathrm{C}_{3} \mathrm{~N}_{4}$ hybrid with enhanced photocatalytic capability under visible light irradiation, J. Mater. Chem. 22 (2012) 2721-2726.

[43] X.G. Duan, H.Q. Sun, Y.X. Wang, J. Kang, S.B. Wang, N-doping-induced nonradical reaction on single-walled carbon nanotubes for catalytic phenol oxidation, ACS Catal. 5 (2015) 553-559.

[44] T. Zhang, Y. Chen, Y. Wang, J. Le Roux, Y. Yang, J.-P. Croué, Efficient peroxydisulfate activation process not relying on sulfate radical generation for water pollutant degradation, Environ. Sci. Technol. 48 (2014) 5868-5875.

[45] H. Lee, H.J. Lee, J. Jeong, J. Lee, N.B. Park, C. Lee, Activation of persulfates by carbon nanotubes: Oxidation of organic compounds by nonradical mechanism, Chem. Eng. J. 266 (2015) 28-33.

[46] Y. Zhou, J. Jiang, Y. Gao, J. Ma, S.Y. Pang, J. Li, X.T. Lu, L.P. Yuan, Activation of peroxymonosulfate by benzoquinone: A novel nonradical oxidation process, Environ. Sci. Technol. 49 (2015) 12941-12950.

[47] Y. Yang, J. Jiang, X.L. Lu, J. Ma, Y.Z. Liu, Production of sulfate radical and hydroxyl radical by reaction of ozone with peroxymonosulfate: A novel advanced oxidation process, Environ. Sci. Technol. 49 (2015) 7330-7339.

[48] Y. Yang, J.J. Pignatello, J. Ma, W.A. Mitch, Comparison of halide impacts on the efficiency of contaminant degradation by sulfate and hydroxyl radical-based advanced oxidation processes (AOPs), Environ. Sci. Technol. 48 (2014) 2344-2351.

[49] J.E. Grebel, J.J. Pignatello, W.A. Mitch, Effect of halide ions and carbonates on organic contaminant degradation by hydroxyl radical-based advanced oxidation processes in saline waters, Environ. Sci. Technol. 44 (2010) 6822-6828.

[50] T. Zhang, H.B. Zhu, J.P. Croue, Production of sulfate radical from peroxymonosulfate induced by a magnetically separable $\mathrm{CuFe}_{2} \mathrm{O}_{4}$ spinel in water: Efficiency, stability, and mechanism, Environ. Sci. Technol. 47 (2013) 2784-2791.

[51] X.Y. Chen, J.W. Chen, X.L. Qiao, D.G. Wang, X.Y. Cai, Performance of nano$\mathrm{Co}_{3} \mathrm{O}_{4} /$ peroxymonosulfate system: Kinetics and mechanism study using Acid Orange 7 as a model compound, Appl. Catal., B 80 (2008) 116-121.

[52] C.D. Qi, X.T. Liu, J. Ma, C.Y. Lin, X.W. Li, H.J. Zhang, Activation of peroxymonosulfate by base: Implications for the degradation of organic pollutants, Chemosphere 151 (2016) 280288. 